Research Article

\title{
Numerical and Computer Simulations of Cross-Flow in the Streamwise Direction through a Moving Surface Comprising the Significant Impacts of Viscous Dissipation and Magnetic Fields: Stability Analysis and Dual Solutions
}

\author{
Yu-Ming Chu $\mathbb{D}^{1,2}$ Umair Khan, ${ }^{3}$ A. Zaib $\mathbb{D}^{4,5}$ and S. H. A. M. Shah ${ }^{3}$ \\ ${ }^{1}$ Department of Mathematics, Huzhou University, Huzhou 313000, China \\ ${ }^{2}$ Hunan Provincial Key Laboratory of Mathematical Modeling and Analysis in Engineering, \\ Changsha University of Science \& Technology, Changsha 410114, China \\ ${ }^{3}$ Department of Mathematics and Social Sciences, Sukkur IBA University, Sukkur 65200, Sindh, Pakistan \\ ${ }^{4}$ Department of Natural Sciences, The Begum Nusrat Bhutto Women University, Sukkur 65170, Pakistan \\ ${ }^{5}$ Department of Mathematical Sciences, Federal Urdu University of Arts Science \& Technology, Gulshan-e-Iqbal, \\ Karachi-75300, Pakistan
}

Correspondence should be addressed to A. Zaib; aurangzaib@fuuast.edu.pk

Received 18 July 2020; Revised 6 September 2020; Accepted 13 September 2020; Published 30 September 2020

Academic Editor: Marin Marin

Copyright (C) 2020 Yu-Ming Chu et al. This is an open access article distributed under the Creative Commons Attribution License, which permits unrestricted use, distribution, and reproduction in any medium, provided the original work is properly cited.

The inspiration for this study is to explore the crucial impact of viscous dissipation (VISD) on magneto flow through a cross or secondary flow (CRF) in the way of streamwise. Utilizing the pertinent similarity method, the primary partial differential equations (PDEs) are changed into a highly nonlinear dimensional form of ordinary differential equations (ODEs). These dimensionless forms of ODEs are executed numerically by the aid of bvp4c solver. The impact of pertinent parameters such as the suction parameter, magnetic parameter, moving parameter, and viscous dissipation parameter is discussed with the help of plots. Dual solutions are obtained for certain values of a moving parameter. The velocities in the direction of streamwise, as well as crossflow, decline in the upper branch solution, while the contrary impact is seen in the lower branch solution. However, the influence of suction on the velocities in both directions uplifts in the upper branch solution and shrinks in the lower branch solution. The analysis is also performed in terms of stability to inspect which solution is stable or unstable, and it is observed that the lower branch solution is unstable, whereas the upper branch one is stable.

\section{Introduction}

The investigations of CRFS started after the pioneering research by Blasius [1] and Prandtl [2] on the laminar flow from a flat surface through a miniature viscosity. Prandtl [3] seemed to be a primary researcher to give the result for the regular depress gradient flow through a yawed infinite cylinder. The study through cross-flow or secondary flow is significant in many engineering applications such as a flow of wind phenomena, mechanical, aerospace, and rotating disk. Jones [4] discussed the vital results involving the problem of CRF, where he scrutinized the influence of sweepback on the boundary layer flow (BOUNLF). The three-dimensional flow past flat surface, as well as curved surfaces, was explored by Mager [5]. Bhattacharyya and Pop [6] examined the dissipation impact on forced convective flow in the way of CRF. Weidman [7] found the outcomes of the flow over an exponentially stretching surface involving the power law at which CRFS is shaped via the activity of the transverse wall shearing. Haq et al. [8] scrutinized the CRF in the direction of streamwise (STW) from a heated moving surface comprising the influence of viscous dissipation (VISD). Itu et al. [9] utilized the finite element technique to improve the rigidity of circular composite surfaces via radial 
ribs. Khan et al. [10] considered the combined impacts of a chemical reaction (CHEMR) and activation energy (ACTIE) comprising nanoparticles through a CRF and STW direction with nonlinear radiation. They reported the dual nature of solutions. Recently, Nisar et al. [11] explored the nonlinear radiation impact on MHD flow of titanium and aluminum alloy particles in the CRF and STW flow direction.

The investigations of the properties of magnetic assets also named magnetohydrodynamics (MHD) flow on electrically conducting liquids conferred its importance in industrial and engineering fields such as heat insulation, geothermal systems, boilers, saltwater, nuclear process, energy storage, and biological transportation. Abbas [12] obtained the equation of frequency for radial vibrations through a poroelastic cylinder in a porous lid by utilizing Biot's theory for wave propagation. Groza and Pop [13] explored the fractional problems of linear multipoint as well as two-point boundary value and obtained the numerical solution by implementing the Haar wavelet technique. Niculita et al. [14] used the finite element technique by utilizing the skin panel for the adaptive wing. Ali [15] found the dual outcomes of magnetoviscous flow through a nonlinear permeable shrinking surface. Mabood et al. [16] analyzed the influence of CHEMR on magnetorotated liquid from a vertical surface entrenched in a permeable medium with a heat source. Kumar et al. [17] scrutinized the frictional heating impact on magneto flow (MAGF) comprising Ferro liquid in the existence of radiation. Bhatti and Rashidi [18] explored the entropy analysis on magnetonanoliquid through a stretching sheet. Abbas and Marin [19] established the generalized thermoelasticity through a pulsed laser and obtained the analytic solution. The stimulus of thermal and exponential space-dependent heat sources on MHD nanoliquid through a rotating disk was observed by Makinde et al. [20]. Riaz et al. [21] considered the impact of bioheat on the 3D flow of non-Newtonian fluid in a peristaltic motion through a rectangular cross-section. Mabood Shateyi [22] deliberated the time-dependent MAGF with heat and mass transport embedded in a porous stretching sheet with radiation. Recently, Ahmad et al. [23] investigated the magnetic field on time-dependent squeezing flow between two horizontal surfaces involving non-Newtonian liquid.

The fluctuating destruction of the velocity gradients through the viscous stresses is signified as viscous dissipation. This fractional irreversible process is frequently referred to as the conversion of kinetic energy into internal energy of the liquid (heating up the liquid owing to viscosity because dissipation is elevated in the regions through great gradients). The concept of VISD was first considered by Brinkman [24]. He utilized the viscous dissipation (VISD) concept in the capillary flows. Gebhart [25] scrutinized the impact of VISD in free convective liquid flows. The stimulus of VISD on free convective flow in the non-Darcy regime was explored by Murthy and Singh [26], and they explored the ten percent decrement in three split regions of fluids. Tunc and Bayazitoglu [27] utilized the technique of integral transform to solve the problem containing viscous dissipation along with the transfer of heat in microtubes. The influences of thermal radiation (THERMR) and VISD on time-dependent magneto flow through a heated infinite vertical surface were inspected by Cookey et al. [28]. Partha et al. [29] discovered the VISD influence on mixed convective flow through an exponential stretched sheet. Ibrahim et al. [30] studied the combined effects of THERMR and VISD on time-dependent flow involving micropolar fluid. The BOUNLF and the transfer rate of heat involving Sisko liquid from an expanded or stretched cylinder through erratic thermal conductivity and VISD were inspected by Malik et al. [31]. Recently, Hussain et al. [32] discussed the impact of VISD on MAGF of a non-Newtonian liquid over a nonlinear heated stretched surface.

As discussed earlier, the problem containing combined effects of the magnetic field and viscous dissipation is not yet explored. Therefore, we are examining the viscous dissipation effect along with the magnetic field in the cross-flow and streamwise directions with the characteristic of heat transport. Another important contribution regarding the problem is the complex dual nature of solutions and the stability analysis which of most researchers missed out. This assessment added an innovative methodology for the researchers, engineers, and scientists to find out the key features of the transfer rate of heat in the STW direction of CRF. The subsequent model is numerically handled using bvp4c software. The effects of the important parameters are contested with the assistance of graphs. The problems involving cross-flow taken here are exceptional in that the transverse motion is believed to be fully developed. In several engineering conditions, the cross-flowing stream may be dependent strongly on the orientation and position of a second leading edge.

\section{Problem Formulation}

The current proposed model is measured using the approach of streamwise (STW) and cross-flow (CRF) directions within the boundary layer. The Cartesian coordinates and their corresponding velocities are symbolically denoted by $(x, y, z)$ and $(u, v, w)$, respectively. Based on the secondary flow phenomena, to deal with the three-dimensional (cross-flow and streamwise directions) flow over a heated surface under the variable applied magnetic field, $B(x)=B_{0} / \sqrt{2 x}$. They accomplish a motion along with an unchanged velocity $-\lambda U_{1}$ into and out of the origin located at a position $x$ equal zero, where the coordinate $x$ is run in the path of a flat surface, while the dimensionless moving parameter and the unchanged velocity are denoted by $\lambda$ and $U_{1}$, respectively. Also, $T_{w}$ and $T_{\infty}$ are the uniform wall and ambient temperature of the liquid, whereas the constant and ambient or free-stream concentration of the liquid are symbolically signified by $C_{w}$ and $C_{\infty}$, respectively. The problem is schematically captured in Figure 1. Also, it is presumed that the CRF has a range that is extended widely in the spanwise direction. So, the components of the momentum, energy, and concentration equation did not depend upon the $z$ coordinate. The exercising of the aforementioned conventions along with the Boussinesq approximation and the BOUNDL scaling the leading PDEs is $[6,8,11]$ 

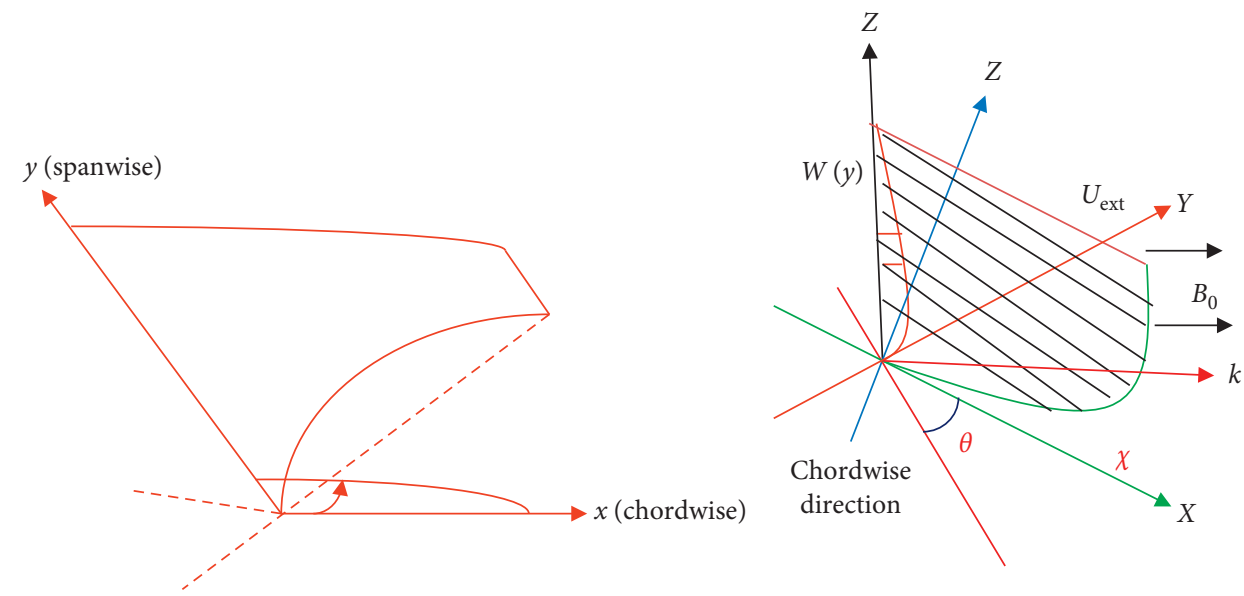

Figure 1: Diagram of the problem.

$$
\begin{aligned}
u_{x}+v_{y} & =0, \\
u u_{x}+v u_{y}-v_{f} u_{y y} & =\frac{\sigma_{f} B^{2}}{\rho_{f}}\left(U_{1}-u\right), \\
u w_{x}+v w_{y}-v_{f} w_{y y} & =\frac{\sigma_{f} B^{2}}{\rho_{f}}\left(w_{1}-w\right), \\
u T_{x}+v T_{y}-\alpha_{f} T_{y y} & =\frac{\mu_{f}}{\left(\rho c_{p}\right)_{f}}\left(\left(u_{y}\right)^{2}+\left(w_{y}\right)^{2}\right), \\
u C_{x}+v C_{y} & =D_{B} C_{y y},
\end{aligned}
$$

with the corresponding boundary conditions

$$
\begin{aligned}
& u(x, 0)=-\lambda U_{1}, \\
& v(x, 0)=v_{0}, \\
& w(x, 0)=0, \\
& T(x, 0)=T_{w}, \\
& C(x, 0)=C_{w}, \\
& u(x, y) \longrightarrow U_{1}, \\
& w(x, y) \longrightarrow w_{1}, \\
& T(x, y) \longrightarrow T_{\infty}, \\
& C(x, y) \longrightarrow C_{\infty} \text { as } y \longrightarrow \infty .
\end{aligned}
$$

Here, the component velocities in the respective directions of $x-, y-$, and $z$-axes are signified by $u(x, y), v(x, y)$, and $w(x, y)$, respectively, while the subscript such as $x$ and $y$ of the velocity components is called the partial derivatives. The other symbols involved in equations (1)-(6) are abbreviated as the dynamic viscosity $\mu_{f}$, density $\rho_{f}$, temperature of the liquid $T$, thermal diffusivity $\alpha_{f}$, effective heat capacity $\left(\rho c_{p}\right)_{f}$, concentration $C$, Brownian diffusion coefficient $D_{B}$, and thermal conductivity $k_{f}$. For simplicity, the technique for solving the problem here by introducing the similarity transformations is as follows:

$$
\begin{aligned}
\eta & =y \sqrt{\frac{U_{1}}{2 x v_{f}}}, \\
\psi & =\sqrt{2 x U_{1} v_{f}} f(\eta), \\
w & =w_{1} g(\eta), \\
\phi(\eta) & =\frac{C_{\infty}-C}{C_{\infty}-C_{w}} \\
\theta(\eta) & =\frac{T_{\infty}-T}{T_{\infty}-T_{w}} .
\end{aligned}
$$

Thus, equation (1) is true identically, while equations (2)-(5) are worked out by the similarity variables. The governing dimensional form of ordinary differential equations become as follows:

$$
\begin{aligned}
\frac{\mathrm{d}^{3} f}{\mathrm{~d} \eta^{3}}+f \frac{\mathrm{d}^{2} f}{\mathrm{~d} \eta^{2}}+M\left(1-\frac{\mathrm{d} f}{\mathrm{~d} \eta}\right) & =0, \\
\frac{\mathrm{d}^{2} g}{\mathrm{~d} \eta^{2}}+f \frac{\mathrm{d} g}{\mathrm{~d} \eta}+M(1-g) & =0, \\
\frac{\mathrm{d}^{2} \theta}{\mathrm{d} \eta^{2}}+\operatorname{Pr} f \frac{\mathrm{d} \theta}{\mathrm{d} \eta}+\operatorname{Pr}\left[E c_{1}\left(\frac{\mathrm{d}^{2} f}{\mathrm{~d} \eta^{2}}\right)^{2}+E c_{2}\left(\frac{\mathrm{d} g}{\mathrm{~d} \eta}\right)^{2}\right] & =0, \\
\frac{\mathrm{d}^{2} \phi}{\mathrm{d} \eta^{2}}+L e f \frac{\mathrm{d} \phi}{\mathrm{d} \eta} & =0 .
\end{aligned}
$$

The converted boundary conditions are

$$
\begin{gathered}
f(\eta)=S, \\
\frac{\mathrm{d} f(\eta)}{\mathrm{d} \eta}=-\lambda, \\
g(\eta)=0,
\end{gathered}
$$




$$
\begin{aligned}
\theta(\eta) & =1, \\
\phi(\eta) & =1, \text { at } \eta=0, \\
\frac{\mathrm{d} f(\eta)}{\mathrm{d} \eta} \longrightarrow 1, & \longrightarrow 1, \\
g(\eta) & \longrightarrow 0, \\
\theta(\eta) & \longrightarrow 0 \text { as } \eta \longrightarrow \infty .
\end{aligned}
$$

In the aforementioned equations, the dimensionless sundry parameters are mathematically expressed as follows: $M=\sigma B_{0}^{2} / \rho_{f} U_{1}, \quad \operatorname{Pr}=v_{f} / \alpha_{f}, \quad L e=\alpha_{f} / D_{B}, \quad E c_{1}=U_{1}^{2} / c_{p}$ $\left(T_{w}-T_{\infty}\right)$, and $E c_{2}=w_{1}^{2} / c_{p}\left(T_{w}-T_{\infty}\right)$ are, respectively, called the magnetic parameter, Prandtl number, Lewis number, and Eckert numbers.

2.1. Skin Friction. The coefficient of skin friction over the STW $\left(C_{f x}\right)$ in the $x$-direction and CRF $\left(C_{f z}\right)$ in the $z$-direction is written as

$$
\begin{aligned}
& C_{f x}=\frac{\mu_{f}\left(u_{y}\right)_{y=0}}{\rho_{f} U_{1}}=\frac{f^{\prime \prime}(0)}{\sqrt{2 \mathrm{Re}_{x}}} \\
& C_{f z}=\frac{\mu_{f}\left(w_{y}\right)_{y=0}}{\rho_{f} w_{1}^{2}}=\frac{g^{\prime}(0)}{\sqrt{2 \operatorname{Re}_{x}}\left(w_{1} / U_{1}\right)} .
\end{aligned}
$$

2.2. Nusselt Number. The local rate of heat transfer in dimensionless form is written as

$$
N u_{x}=\frac{x\left(-k_{f} T_{y}\right)_{y=0}}{k_{f}\left(T_{w}-T_{\infty}\right)}=-\frac{\theta^{\prime}(0)}{\sqrt{2 \mathrm{Re}_{x}}} .
$$

2.3. Sherwood Number. The local Sherwood number in dimensionless form is written as

$$
S h_{x}=\frac{x\left(-C_{y}\right)_{y=0}}{D_{f}\left(C_{w}-C_{\infty}\right)}=-\frac{\phi^{\prime}(0)}{\sqrt{2 \mathrm{Re}_{x}}},
$$

where the correlation of the Reynolds number is $\operatorname{Re}_{x}=x U_{1} / \nu_{f}$.

\section{Stability Analysis}

Within this section, the linear stability analysis of the solutions achieved is carried out to test their stability. Because of such purpose, we cited the work concluded through the study of Merkin [33]. Considering the time-dependent form of equations (2)-(5) along with $\mathrm{BC}(6)$, we may write

$$
\begin{gathered}
u_{t}+u u_{x}+v u_{y}-v_{f} u_{y y}=\frac{\sigma B^{2}}{\rho_{f}}\left(U_{1}-u\right), \\
w_{t}+u w_{x}+v w_{y}-v_{f} w_{y y}=\frac{\sigma B^{2}}{\rho_{f}}\left(w_{1}-w\right), \\
T_{t}+u T_{x}+v T_{y}-\alpha_{f} T_{y y}=\frac{\mu_{f}}{\left(\rho c_{p}\right)_{f}}\left(\left(u_{y}\right)^{2}+\left(w_{y}\right)^{2}\right), \\
C_{t}+u C_{x}+v C_{y}=D_{B}\left(C_{y y}\right),
\end{gathered}
$$

subject to the boundary conditions

$$
\begin{aligned}
& u(x, 0, t)=-\lambda U_{1}, \\
& v(x, 0, t)=v_{0}, \\
& w(x, 0, t)=0, \\
& T(x, 0, t)=T_{w}, \\
& C(x, 0, t)=C_{w}, \\
& u(x, y, t) \longrightarrow U_{1}, \\
& w(x, y, t) \longrightarrow w_{1}, \\
& T(x, y, t) \longrightarrow T_{\infty}, \\
& C(x, y, t) \longrightarrow C_{\infty} \text { as } y \longrightarrow \infty .
\end{aligned}
$$

By letting the fresh time-dependent similarity variable $\tau$, the old transformations (7) may be in mathematical form as follows:

$$
\begin{aligned}
\eta & =y \sqrt{\frac{U_{1}}{2 x v_{f}}}, \\
\psi & =\sqrt{2 x U_{1} v_{f}} f(\eta), \\
w & =w_{1} g(\eta), \\
\theta(\eta) & =\frac{T-T_{\infty}}{T_{w}-T_{\infty}}, \\
\phi(\eta) & =\frac{C-C_{\infty}}{C_{w}-C_{\infty}},
\end{aligned}
$$

where $\tau=U_{1} t / 2 x$ and $\eta=y \sqrt{U_{1} / 2 x v_{f}}$.

Using (21), equations (16)-(19) along with the appropriate BC (20) reduce to the simplest form as follows:

$$
\begin{aligned}
& \frac{\partial^{3} f(\eta, \tau)}{\partial \eta^{3}}+\left(2 \tau \frac{\partial f(\eta, \tau)}{\partial \eta} \frac{\partial^{2} f(\eta, \tau)}{\partial \tau \partial \eta}-\frac{\partial^{2} f(\eta, \tau)}{\partial \tau \partial \eta}\right) \\
& +f(\eta, \tau) \frac{\partial^{2} f(\eta, \tau)}{\partial \eta^{2}}+\frac{\sigma_{f} B_{0}^{2}}{\rho_{f} U_{1}}\left(1-\frac{\partial f(\eta, \tau)}{\partial \eta}\right)=0,
\end{aligned}
$$




$$
\begin{gathered}
\frac{\partial^{2} g(\eta, \tau)}{\partial \eta^{2}}+\left(2 \tau \frac{\partial f(\eta, \tau)}{\partial \eta} \frac{\partial g(\eta, \tau)}{\partial \tau}-\frac{\partial g(\eta, \tau)}{\partial \tau}\right) \\
+f(\eta, \tau) \frac{\partial g(\eta, \tau)}{\partial \eta}+\frac{\sigma_{f} B_{0}^{2}}{\rho_{f} U_{1}}(1-g(\eta, \tau))=0, \\
\frac{1}{\operatorname{Pr}} \frac{\partial^{2} \theta(\eta, \tau)}{\partial \eta^{2}}+E c_{1}\left(\frac{\partial^{2} f(\eta, \tau)}{\partial \eta^{2}}\right)^{2}+f(\eta, \tau) \frac{\partial \theta(\eta, \tau)}{\partial \eta} \\
+E c_{2}\left(\frac{\partial g(\eta, \tau)}{\partial \eta}\right)^{2}-\frac{\partial \theta(\eta, \tau)}{\partial \tau}=0, \\
\frac{\partial^{2} \phi(\eta, \tau)}{\partial \eta^{2}}+\operatorname{Lef}(\eta, \tau) \frac{\partial \phi(\eta, \tau)}{\partial \eta}-\frac{\partial \phi(\eta, \tau)}{\partial \tau}=0,
\end{gathered}
$$

along with the corresponding BCs

$$
\begin{aligned}
& f(\eta, \tau)=S, \\
& \frac{\partial f(\eta, \tau)}{\partial \eta}=-\lambda, \\
& g(\eta, \tau)=0, \\
& \theta(\eta, \tau)=1, \\
& \phi(\eta, \tau)=1 \text { at } \eta=0, \\
& \frac{\partial f(\eta, \tau)}{\partial \eta} \longrightarrow 1, \\
& g(\eta, \tau) \longrightarrow 1, \\
& \theta(\eta, \tau) \longrightarrow 0, \\
& \phi(\eta, \tau) \longrightarrow 0 \text { as } \eta \longrightarrow \infty .
\end{aligned}
$$

The key physical dimensional constraints involved in the above equations are the same and are defined earlier. The stability analysis of the free time-dependent solution $f(\eta, 0)=f_{0}(\eta)$ and $g(\eta, 0)=g_{0}(\eta)$ for the momentum equation in both directions and further $\theta(\eta, 0)=\theta_{0}(\eta)$ and $\phi(\eta, 0)=\phi_{0}(\eta)$ signify the corresponding energy and concentration equations. Let

$$
\begin{aligned}
& f(\eta, \tau)=f_{0}(\eta)+e^{-\beta t} F(\eta, \tau), \\
& g(\eta, \tau)=g_{0}(\eta)+e^{-\beta t} G(\eta, \tau), \\
& \theta(\eta, \tau)=\theta_{0}(\eta)+e^{-\beta t} \Theta(\eta, \tau), \\
& \phi(\eta, \tau)=\phi_{0}(\eta)+e^{-\beta t} \Phi(\eta, \tau),
\end{aligned}
$$

where $\beta$ highlights the eigenvalues. Using (27) into (22)-(26), we may achieve the following linearized eigenvalue problem:

$$
\begin{gathered}
F^{\prime \prime \prime}+f_{0} F^{\prime \prime}+F f_{0}^{\prime \prime}+\beta F^{\prime}-M F^{\prime}=0, \\
G^{\prime \prime}+f_{0} G^{\prime}+F g_{0}^{\prime}+\beta G-M G=0,
\end{gathered}
$$

$$
\Theta^{\prime \prime}+\operatorname{Pr}\left[f_{0} \Theta^{\prime}+F \theta_{0}^{\prime}+E c_{1}\left(2 f_{0}^{\prime} F^{\prime \prime}\right)^{2}+E c_{2}\left(2 g_{0}^{\prime} G^{\prime}\right)^{2}+\beta \Theta\right]=0,
$$

$$
\Phi^{\prime \prime}+\operatorname{Le}\left(f_{0} \Phi^{\prime}+F \phi_{0}^{\prime}\right)+\beta \Phi=0 .
$$

along with the appropriate BCs

$$
\begin{aligned}
& F(\eta)=0, \\
& F^{\prime}(\eta)=0, \\
& G(\eta)=0, \\
& \Theta(\eta)=0, \\
& \Phi(\eta)=0 \text { at } \eta=0, \\
& F^{\prime}(\eta) \longrightarrow 0, \\
& G(\eta) \longrightarrow 0, \\
& \Theta(\eta) \longrightarrow 0, \\
& \Phi(\eta) \longrightarrow 0 \text { at } \eta=\infty
\end{aligned}
$$

The eigenvalue outcome of the linearized-type problem provides the class of eigenvalues. Because of the approach of linear stability analysis, the initial deceleration of disturbance, which is a physically reliable outcome (stable), is observed because of the positive coarse eigenvalue, while the initial development of disturbance is occurred owing to the negative coarse values which provide the unstable outcome. Furthermore, it is easy to note all of the positive smallest eigenvalues which indicates that the results achieved are stable and physically consistent. Therefore, in the current problem, equations (28)-(32) are solved for the eigenvalue $\beta$ with the new BC $F^{\prime \prime}=1$ by relaxing the condition that $F^{\prime} \longrightarrow 0$ as $\eta \longrightarrow \infty$.

\section{Results and Discussion}

In this current section, the numerical results of the streamwise and cross-flow problem governed by the set of nonlinear partial differential equations (PDEs) are presented. To solve the nonlinear PDEs (2)-(5), the similarity variables are first introduced to transform the problem into a system of highly nonlinear ODEs (8)-(11). These ODEs are very difficult to solve exactly; therefore, it can be tackled numerically by using the scheme of finite difference for boundary value problem based on the three-stage Lobatto IIIA. For the computations, the fixed values assigned to the parameters are $S=L e=0.5, \lambda=-3.5, M=0.1, E c_{1}=0.1$, $E c_{2}=0.2$, and $\operatorname{Pr}=6.2$. The dual or multiple solutions of the problem under consideration are obtained for the specific range of the stretching and shrinking constraints. In our results, the upper branch solution and the lower branch solution is depicted graphically with the solid and dotted lines, respectively.

4.1. Effects of Magnetic Parameter. The influence of the magnetic parameter $M$ is captured in the STW and CRF directions in Figures 2(a) and 2(b), respectively. The 


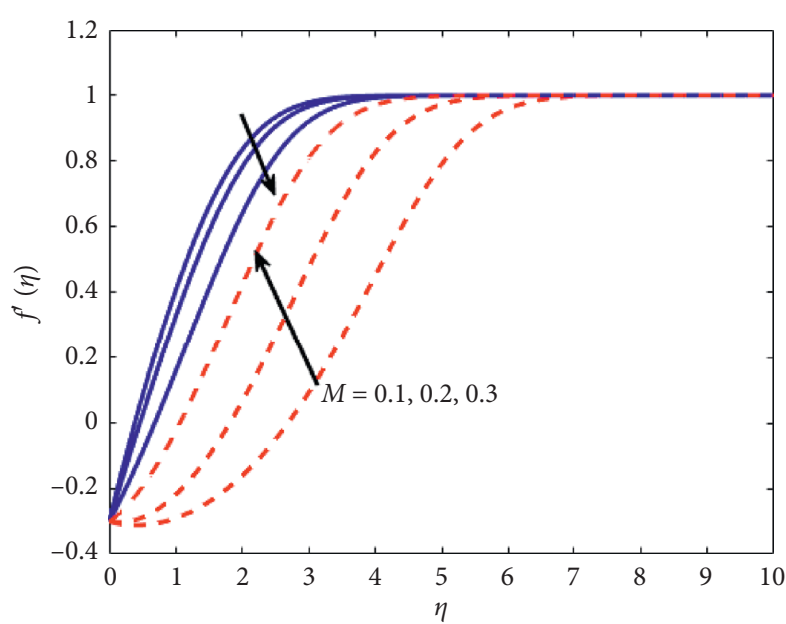

(a)

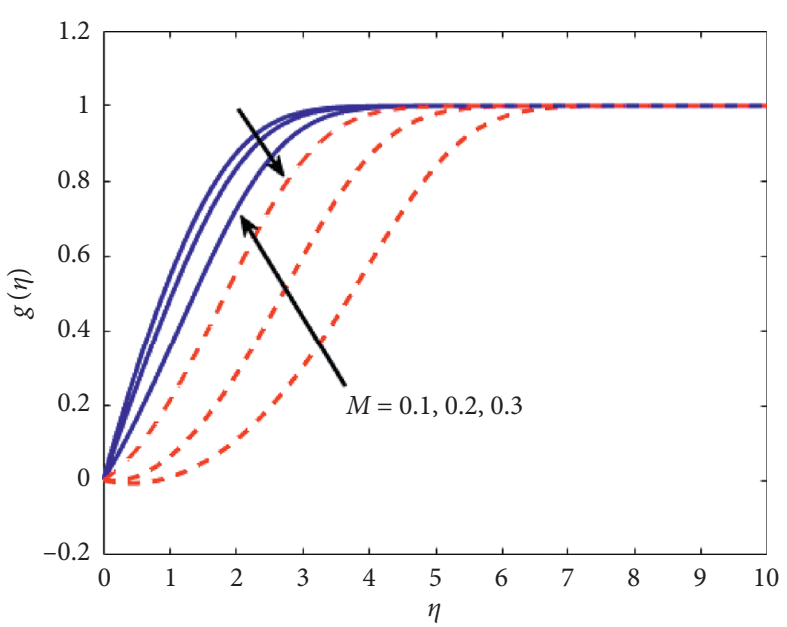

(b)

Figure 2: (a, b) Impact of $M$ on $f^{\prime}(\eta)$ and $g(\eta)$.

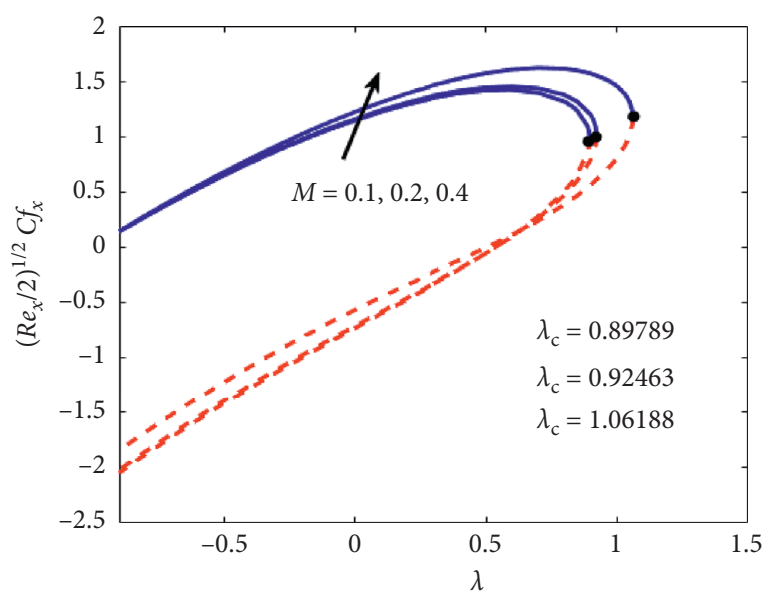

(a)

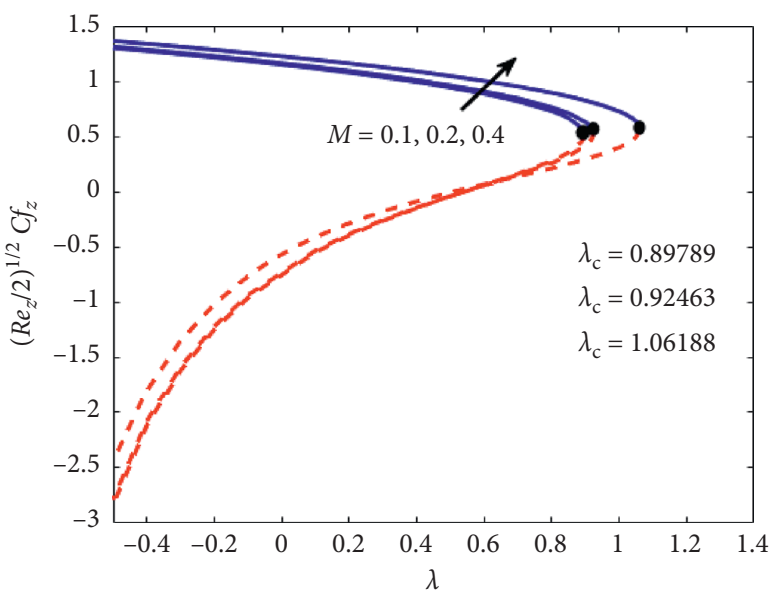

(b)

Figure 3: $(\mathrm{a}, \mathrm{b})$ Impact of $M$ versus $\lambda$ on $\left(2 \mathrm{Re}_{x}\right)^{1 / 2} C f_{x}$ and $\left(2 \operatorname{Re}_{x}\right)^{1 / 2} C f_{z}$.

solutions obtained for $f^{\prime}(\eta)$ and $g(\eta)$ satisfy the initial and boundary conditions. The velocity profiles in the STW and $\mathrm{CRF}$ directions decrease in the first branch solution and increase in the second branch solution. Physically, this is happening due to a larger amount of $M$ which can slow down the liquid motion; this occurs due to retarding force known as Lorentz force. Figures 3(a) and 3(b) explain that the skin friction in both directions increases owing to the constraint $M$ in the first branch solution, while the changed behavior is observed in the second branch solution. On the other hand, the rate of heat and mass transfer decreases in the first branch solution and enhances the second branch solution as portrayed in Figures 4(a) and 4(b), respectively. The huge magnetic limit develops the force called drag which is also entitled as Lorentz force. This force controls as mediators that resist the fluid flow and craft progress in the local transfer rate of heat and mass along with the local coefficient of skin coefficient.
4.2. Influence of Suction Parameter. In the porous medium, the mass suction/injection parameter is important and is defined with the expression $S=-v_{0} \sqrt{2 x / v_{f} U_{1}}$. In case of suction, $(S>0)$ and for injection, $(S<0)$. If $(S<0)$, the dual nature of solutions cannot be obtained. However, the dual solutions exist in the case of mass suction. In Figures 5(a) and 5(b), the variation of the suction parameter $S$ on both the velocity profiles in the STW direction in terms of $f^{\prime}(\eta)$ and the CRF in terms of $g(\eta)$ is displayed. These results suggest that the velocity of liquid augments due to increasing values of $S$ in the upper branch solution which in turn reduces the velocity BOUNLF thickness, while there is a decline in the lower branch solution in the STW and CRF directions. Generally, because of $S$, the heated liquid is shoved toward a surface of the wall where the forces can retard the liquid owing to lofty control of the viscosity. The skin friction coefficients in both directions are displayed in Figures 6(a) and 6(b). These figures show that the 


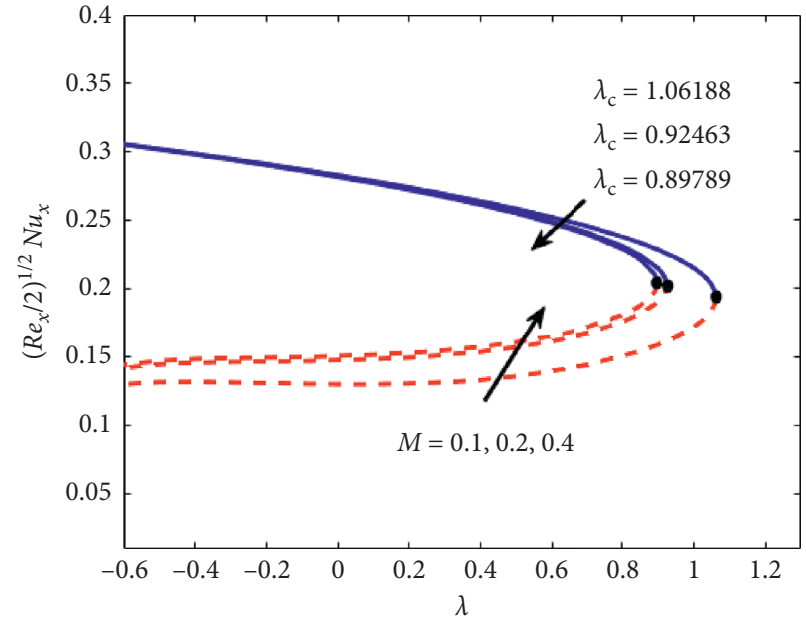

(a)

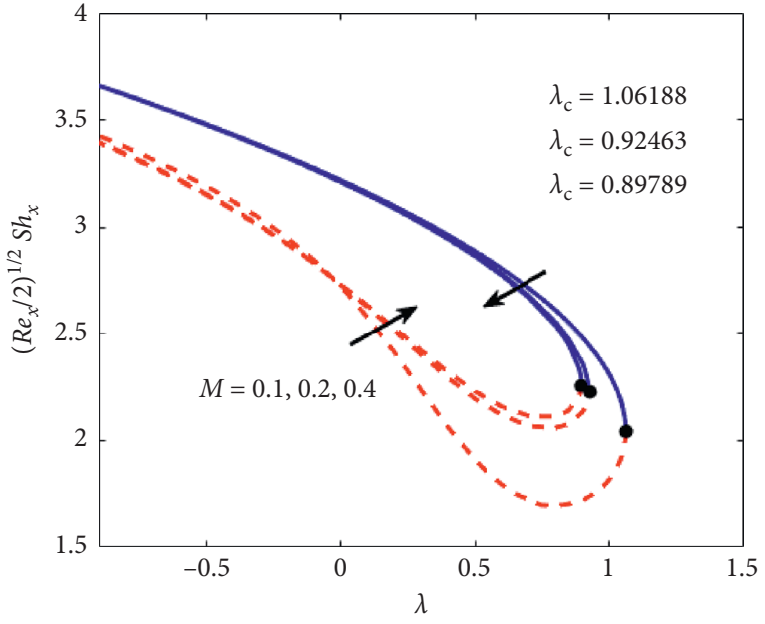

(b)

Figure 4: $(\mathrm{a}, \mathrm{b})$ Impact of $M$ versus $\lambda$ on $\left(2 \mathrm{Re}_{x}\right)^{1 / 2} N u_{x}$ and $\left(2 \mathrm{Re}_{x}\right)^{1 / 2} S h_{x}$.

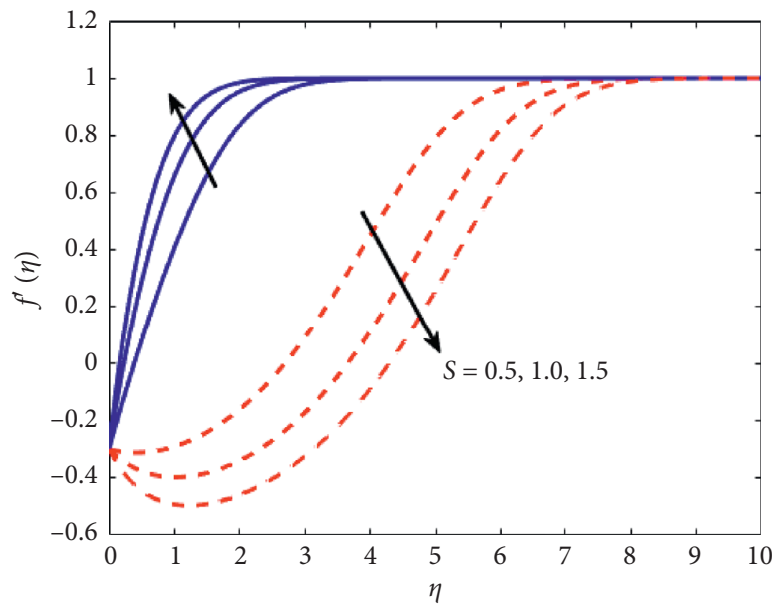

(a)

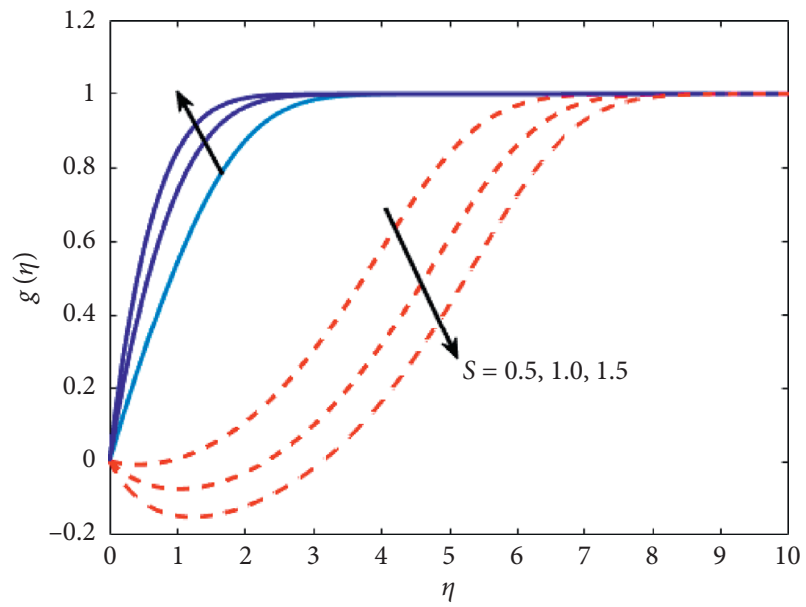

(b)

Figure 5: (a, b) Impact of $S$ on $f^{\prime}(\eta)$ and $g(\eta)$.

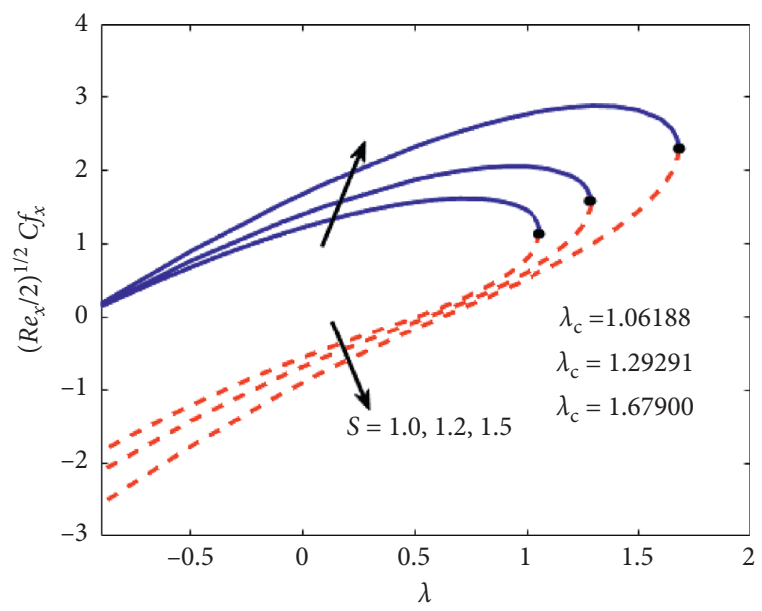

(a)

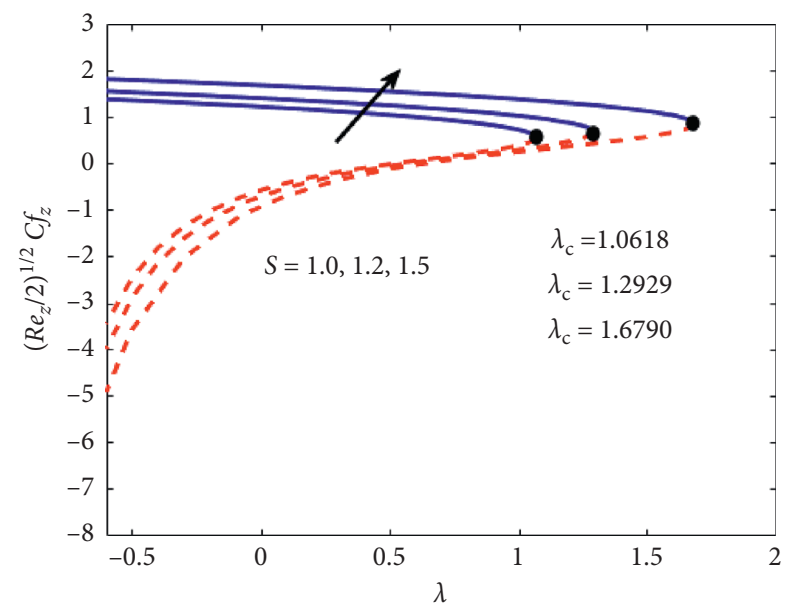

(b)

Figure 6: $(\mathrm{a}, \mathrm{b})$ Impact of $S$ versus $\lambda$ on $\left(2 \mathrm{Re}_{x}\right)^{1 / 2} C f_{x}$ and $\left(2 \mathrm{Re}_{x}\right)^{1 / 2} C f_{z}$. 


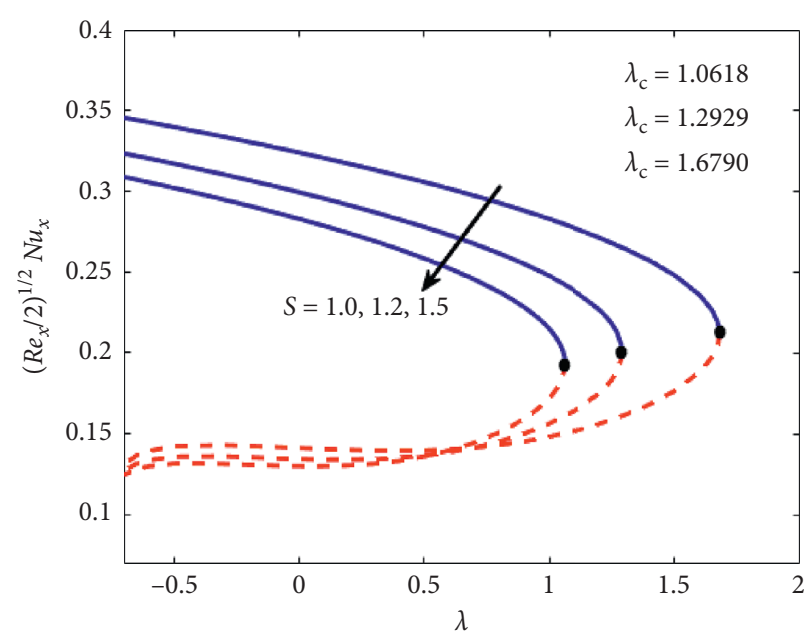

(a)

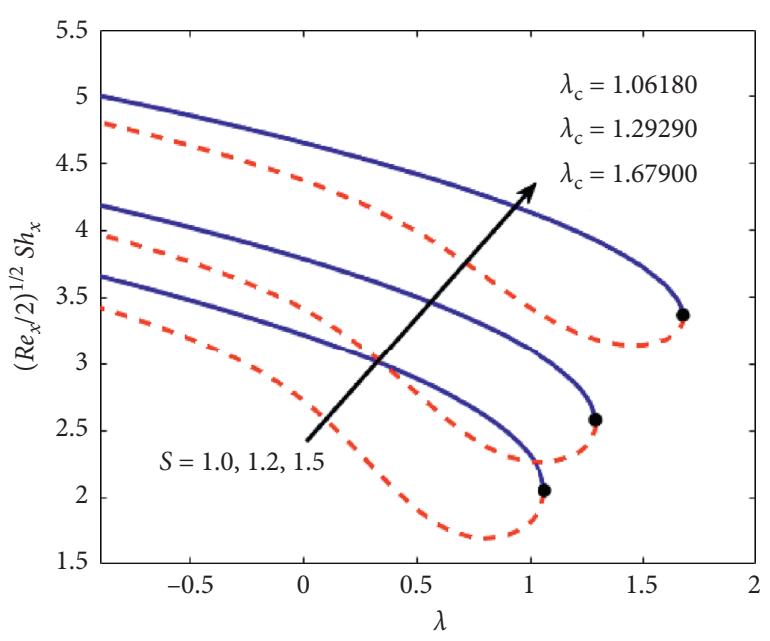

(b)

Figure 7: $(\mathrm{a}, \mathrm{b})$ Impact of $S$ versus $\lambda$ on $\left(2 \operatorname{Re}_{x}\right)^{1 / 2} N u_{x}$ and $\left(2 \operatorname{Re}_{x}\right)^{1 / 2} S h_{x}$.

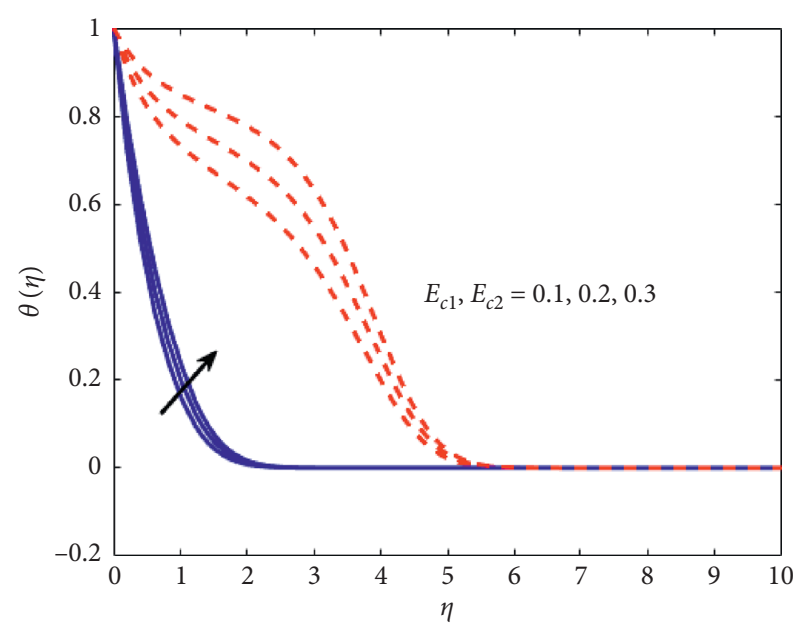

(a)

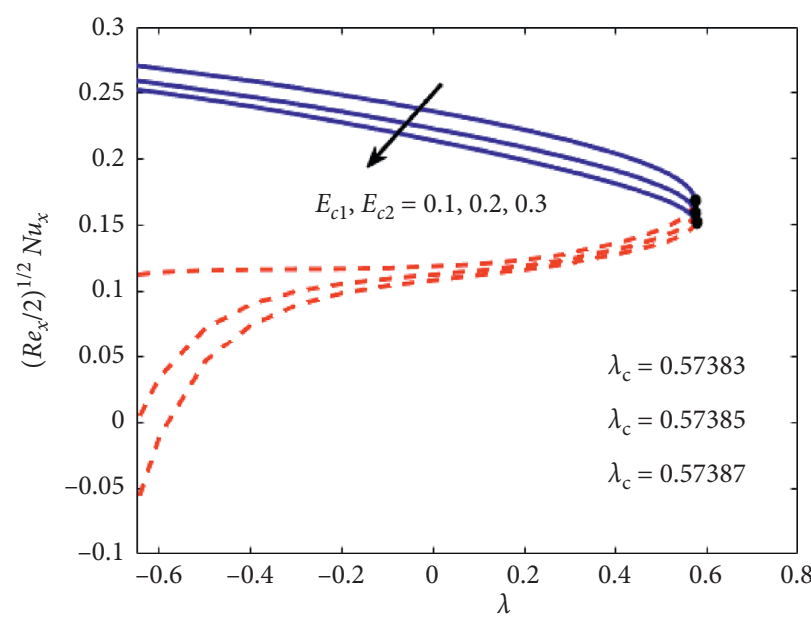

(b)

FIgURE 8: $(\mathrm{a}, \mathrm{b})$ Impact of $E_{c 1}$ and $E_{c 2}$ on $\theta(\eta)$ versus $\lambda$ on $\left(2 \mathrm{Re}_{x}\right)^{1 / 2} N u_{x}$.

upper branch solution increases while the second branch result decreases as the mass suction parameter increases. The variation of $S$ on the local transfer rate of heat and mass is presented in Figures 7(a) and 7(b), respectively. Figure 7(a) depicts the values of the local rate of heat transfer decline due to augmenting $S$ in both branches of solutions, whereas the Sherwood number augments with $S$ in the upper and lower branch solutions as revealed in Figure $7(\mathrm{~b})$.

\subsection{Effects of Eckert Numbers. The Eckert parameters appear} in the energy equation due to the viscous dissipation term. The Eckert numbers have a direct impact on the rate of heat transfer. As the Eckert numbers upsurge, more heat is transferred from the surface due to which the thermal boundary layer increases. This impacts of $E_{c 1}$ and $E_{c 2}$ on temperature $\theta(\eta)$ is displayed in Figure $8(\mathrm{a})$. However, the increases in Eckert numbers show a decrease in Nusselt number (Figure $8(\mathrm{~b})$ ). This is predictable since the implementation of the Eckert number proposes the ratio of kinetic energy and enthalpy. Thus, an augment in $E_{c 1}$ and $E_{c 2}$ suggests that the heat dissipated is stored in the liquid via fractional heating that enhances the liquid temperature and consequently declines the heat transfer rate.

4.4. Effects of Lewis Number. Lewis number $L_{e}$ appears in the concentration equation and is a ratio of the kinematic viscosity and the Brownian diffusion coefficient. The influence of $L_{e}$ on concentration $\phi(\eta)$ is demonstrated in Figure 9(a). The graphical result shows that the concentration profile decreases for both solutions. This implies that the heat will disperse more quickly than concentration. The concentration boundary layer becomes steeper as $L_{e}$ enhances. Moreover, the increase in $L_{e}$ shows an increase in the Sherwood number (Figure 9(b)). 


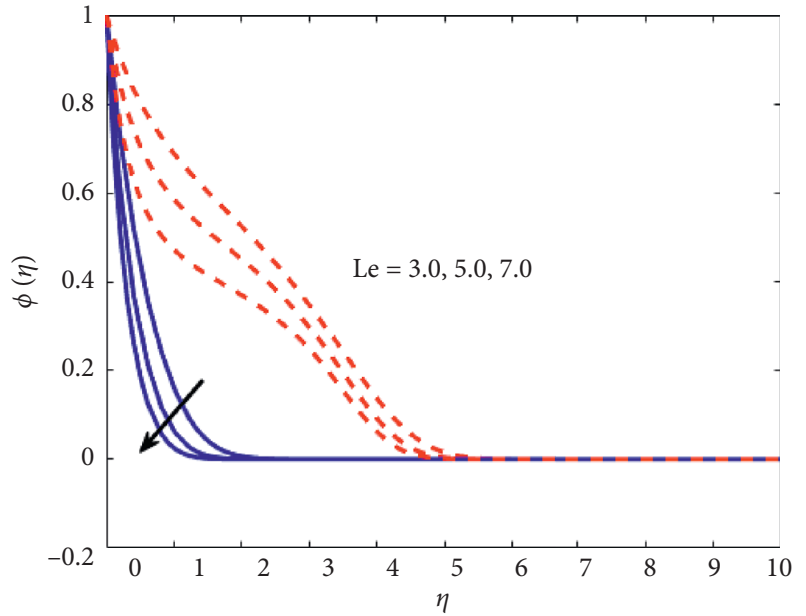

(a)

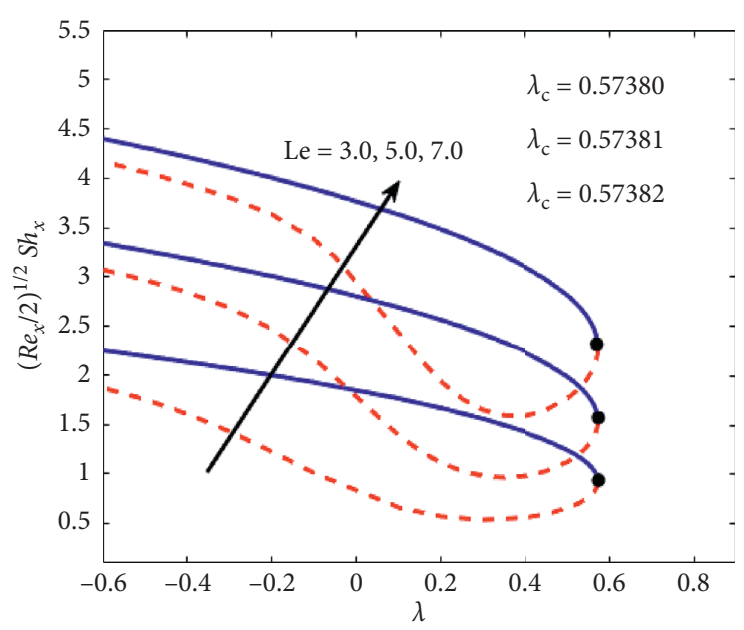

(b)

Figure 9: $(\mathrm{a}, \mathrm{b})$ Impact of $L_{e}$ on $\phi(\eta)$ versus $\lambda$ on $\left(2 \mathrm{Re}_{x}\right)^{1 / 2} S h_{x}$.

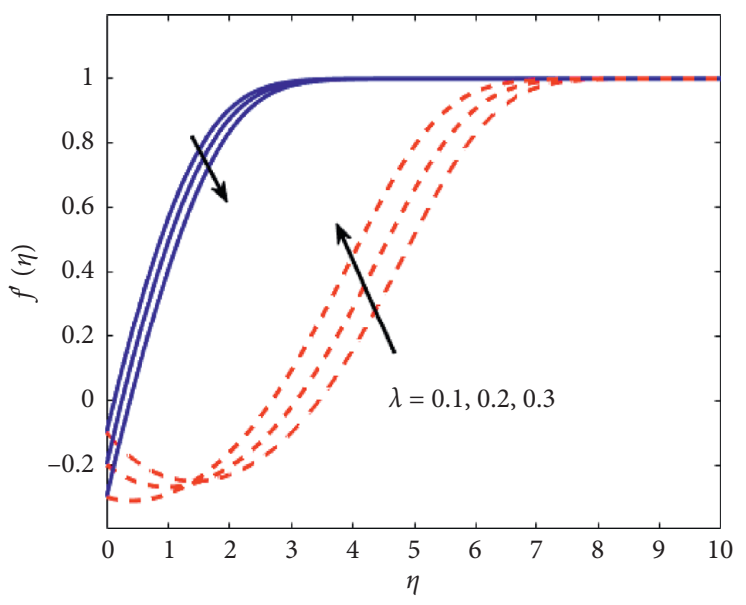

(a)

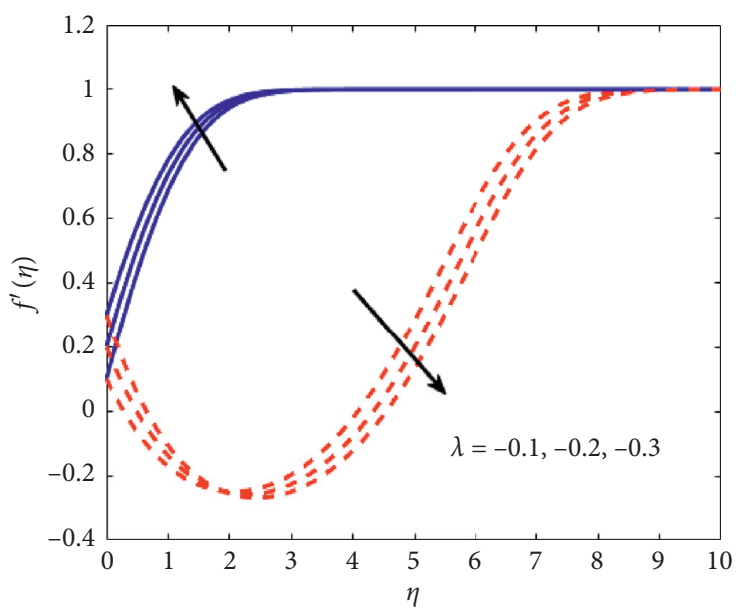

(c)

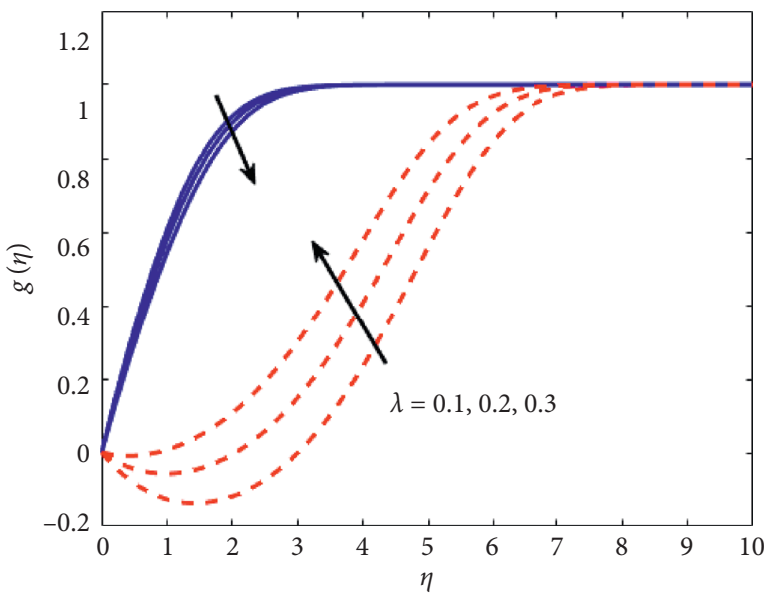

(b)

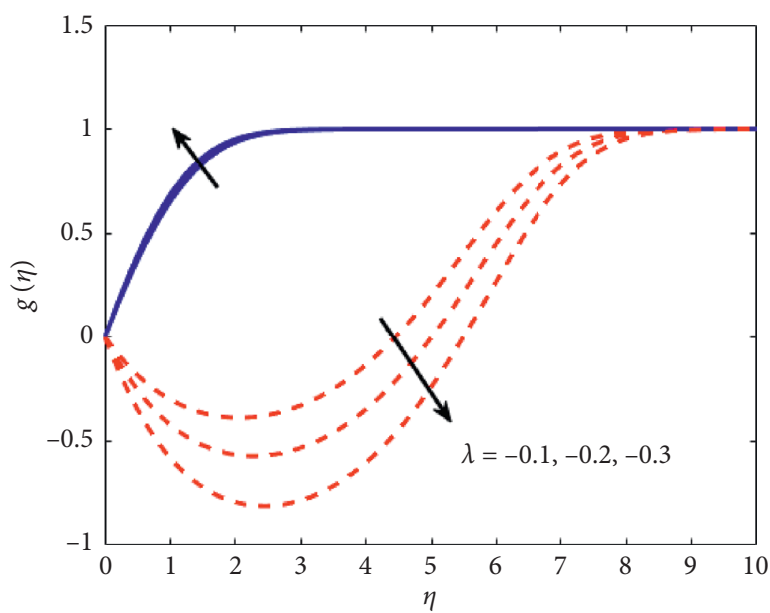

(d)

Figure 10: (a-d) Impact of $\lambda$ on $f^{\prime}(\eta)$ and $g(\eta)$. 
4.5. Effects of Stretching and Shrinking Parameters. In Figures 10(a)-10(d), the variations of stretching/shrinking parameters on the velocities $f^{\prime}(\eta)$ and $g(\eta)$ in both directions are displayed. The effect of the stretching parameter (Figures 10(a) and 10(b)) shows that the lower branch solution decreases while the upper branch solution increases. On the other hand, the altered behavior is noted for the shrinking parameter, as illustrated in Figures $10(\mathrm{c})$ and $10(\mathrm{~d})$. It is also examined from these figures that, in the phenomenon of the lower branch solution, initially, the velocity profiles are negative, and then as the value of $\eta$ progresses, it starts to augment and then becomes positive. Physically, this trend happens due to the contrary directions of shrinking and stretching velocities.

\section{Conclusions}

In the current investigation, the impact of VISD on MAGF near a stagnation point in the directions of STW and CRF through a moving surface is scrutinized. The leading PDEs are converted into nonlinear ODEs via suitable variables. These converted ODEs are worked out by utilizing bvp4c software based on 3-stage Lobatto IIIA. The influences of pertinent constraints on the flow fields such as concentration, velocity, and temperature are illustrated graphically and numerically scrutinized. The core finding of this exploration can be precised as follows:

(i) Because of some amount of moving parameter, multiple solutions are achieved

(ii) The velocity of liquid decays owing to $M$ in the upper branch solution and upsurges in the lower branch solution in the STW and CRF directions

(iii) The friction factor in the STW and CRF directions augments because of the larger value of $M$ in the upper branch solution, while the rate of mass and heat transfer decelerates in the upper branch solution and augments in the lower branch solution

(iv) The mass suction parameter enhances the velocity and friction factor in the upper branch solution and drops in the lower branch solution, whereas the rate of heat transfer declines and the Sherwood number upsurges in the first and second branch solutions

(v) The temperature distribution enhances due to the Eckert number, while the transfer rate of heat declines due to the Eckert number in the upper and lower branch solutions

(vi) The concentration profile decelerates with increasing values of $L_{e}$ in the first branch (upper) solution and the second branch (lower) solutions

(vii) The values of the Sherwood number uplift in both branches of solutions due to $L_{e}$

Finally, this paper can be expanded by taking a timedependent flow or mixed convective flow. Also, the nonNewtonian fluid or hybrid nanofluid may be added due to numerous applications.

\section{Abbreviations}

\begin{tabular}{|c|c|}
\hline$B_{0}:$ & Magnetic field intensity \\
\hline$C:$ & Concentration \\
\hline$C_{\infty}:$ & Ambient fluid concentration \\
\hline$C_{w}:$ & Wall concentration \\
\hline$C_{f x}$ and $C_{f z}$ : & Skin friction coefficients \\
\hline$D_{B}:$ & Brownian diffusion coefficient \\
\hline$E c_{1}$ and $E c_{2}:$ & Eckert numbers \\
\hline$f$ and $g:$ & Dimensionless velocities \\
\hline$k_{f}:$ & Thermal conductivity \\
\hline$L_{e}:$ & Lewis number \\
\hline$M:$ & Magnetic parameter \\
\hline$N u_{x}:$ & Nusselt number \\
\hline Pr: & Prandtl number \\
\hline$R_{d}:$ & Radiation parameter \\
\hline $\operatorname{Re}_{x}$ & Local Reynolds number \\
\hline$S:$ & Suction \\
\hline$S h_{x}:$ & Sherwood number \\
\hline$T:$ & Temperature \\
\hline$T_{\infty}:$ & Free-stream temperature \\
\hline$T_{w}:$ & Temperature of wall \\
\hline$U_{1}:$ & Constant velocity \\
\hline$w_{0}:$ & Transverse velocity \\
\hline$(u, v, w):$ & Velocity components \\
\hline$(x, y, z)$ & Cartesian coordinates \\
\hline$\alpha_{f}:$ & Thermal diffusivity \\
\hline$\beta:$ & Eigenvalues \\
\hline$\lambda:$ & Moving parameter \\
\hline$\mu_{f}:$ & Dynamic viscosity \\
\hline$\rho_{f}:$ & Density \\
\hline$\left(\rho c_{p}\right)_{f}:$ & Effective heat capacity \\
\hline$\theta:$ & Dimensionless temperature \\
\hline$v_{f}:$ & Kinematic viscosity \\
\hline$\psi:$ & Stream function \\
\hline$\tau:$ & Time-dependent similarity variable \\
\hline ': & Derivative w. r. t. $\eta$. \\
\hline
\end{tabular}

\section{Data Availability}

No data were used to support this study.

\section{Conflicts of Interest}

The authors declare that they have no conflicts of interest.

\section{Acknowledgments}

This research was supported by the National Natural Science Foundation of China (Grant nos. 11971142, 11871202, 61673169, 11701176, 11626101, and 11601485).

\section{References}

[1] H. Blasius, "Grenzschichten in flüssigkeiten mit kleiner reibung," Zeitschrift für angewandte Mathematik und Physik, vol. 56, pp. 1-37, 1908.

[2] L. Prandtl, "Uber flussigkeitsbewegung bei sehr kleiner reibung," in Interactive Mathematics.Kongregate, San Francisco, CA, USA, 1904. 
[3] L. Prandtl, "On boundary layers in three-dimensional flow," Aeronautical Research Council, vol. 9829, 1946.

[4] R. T. Jones, "Effects of sweepback on boundary-layer and separation," Technical report, National Advisory Committee 884 NTRS - NASA Technical Reports USA AmesAeronautical Laboratray, Moffett Field, CA, USA, 1947.

[5] A. Mager, "Three-dimensional laminar boundary layer with small cross-flow," Journal of the Aeronautical Sciences, vol. 21, no. 12, pp. 835-845, 1954.

[6] K. Bhattacharyya and I. Pop, "Heat transfer for boundary layers with cross flow," Chinese Physics B, vol. 23, no. 2, Article ID 024701, 2014.

[7] P. Weidman, "Similarity solutions for power-law and exponentially stretching plate motion with cross flow," International Journal of Non-Linear Mechanics, vol. 89, pp. 127-131, 2017.

[8] R. U. Haq, F. A. Soomro, Z. H. Khan, and Q. M. Al-Mdallal, "Numerical study of streamwise and cross flow in the presence of heat and mass transfer," European Physical Journal Plus, vol. 132, no. 5, p. 214, 2017.

[9] C. Itu, A. Öchsner, S. Vlase, and M. I. Marin, "Improved rigidity of composite circular plates through radial ribs," Proceedings of the Institution of Mechanical Engineers, Part L: Journal of Materials: Design and Applications, vol. 233, no. 8, pp. 1585-1593, 2019.

[10] U. Khan, A. Zaib, I. Khan, and K. S. Nisar, "Activation energy on MHD flow of titanium alloy (Ti6Al4V) nanoparticle along with a cross flow and streamwise direction with binary chemical reaction and non-linear radiation: Dual Solutions," Journal of Materials Research and Technology, vol. 9, no. 1, pp. 188-199, 2019.

[11] K. S. Nisar, U. Khan, A. Zaib, I. Khan, and D. Baleanu, "Exploration of aluminum and titanium alloys in the streamwise and secondary flow directions comprising the significant impacts of magnetohydrodynamic and hybrid nanofluid," Crystals, vol. 10, no. 8, p. 679, 2020.

[12] I. A. Abbas, "Natural frequencies of a poroelastic hollow cylinder," Acta Mechanica, vol. 186, no. 1-4, pp. 229-237, 2006.

[13] G. Groza and N. Pop, "A numerical method for solving of the boundary value problems for ordinary differential equations," Results in Mathematics, vol. 53, no. 3-4, pp. 295-302, 2012.

[14] C. Niculita, S. Vlase, A. Bencze, M. Mihalcica, M. R. Calin, and L. Serbina, "Optimum stacking in a multi-ply laminate used for the skin of adaptive wings, Optoelect," Advanced MaterialRapid Communications, vol. 5, no. 11, pp. 1233-1236, 2011.

[15] F. M. Ali, R. Nazar, N. M. Arifin, and I. Pop, "Dual solutions in MHD flow on a nonlinear porous shrinking sheet in a viscous fluid," Boundary Value Problems, vol. 2013, no. 1, 2013.

[16] F. Mabood, S. M. Ibrahim, and G. Lorenzini, "Chemical reaction effects on MHD rotating fluid over a vertical plate embedded in porous medium with heat source," Journal of Engineering Thermophysics, vol. 26, no. 3, pp. 399-415, 2017.

[17] K. A. Kumar, V. Ramana Reddy Janke, S. Vangala, and S. Naramgari, "Impact of frictional heating on MHD radiative ferrofluid past a convective shrinking surface," Defect Diffusion Forum, vol. 378, pp. 157-174, 2017.

[18] M. M. Bhatti and M. M. Rashidi, "Numerical simulation of entropy generation on MHD nanofluid towards a stagnation point flow over a stretching surface," International Journal of Applied and Computational Mathematics, vol. 3, no. 3, pp. 2275-2289, 2017.
[19] I. A. Abbas and M. Marin, "Analytical solution of thermoelastic interaction in a half-space by pulsed laser heating," Physica E: Low-Dimensional Systems and Nanostructures, vol. 87 , pp. 254-260, 2017.

[20] O. D. Makinde, B. Mahanthesh, B. J. Gireesha, N. S. Shashikumar, R. L. Monaledi, and M. S. Tshehla, "MHD nanofluid flow past a rotating disk with thermal radiation in the presence of aluminum and titanium alloy nanoparticles," Defect and Diffusion Forum, vol. 384, pp. 69-79, 2018.

[21] A. Riaz, R. Ellahi, M. M. Bhatti, and M. Marin, "Study of heat and mass transfer in the eyring-powell model of fluid propagating peristaltically through a rectangular compliant channel," Heat Transfer Research, vol. 50, no. 16, pp. 1539-1560, 2019.

[22] F. Mabood and S. Shateyi, "Multiple slip effects on MHD unsteady flow heat and mass transfer impinging on permeable stretching sheet with radiation," Modelling and Simulation in Engineering, vol. 2019, pp. 1-11, 2019.

[23] S. Ahmad, M. Farooq, A. Anjum, and S. Sheriff, "Investigation of convective heat and mass conditions in squeeze flow of a hydro-magnetic sutterby fluid," Journal of Magnetics, vol. 24, no. 4, pp. 688-697, 2019.

[24] H. C. Brinkman, "Heat effects in capillary flow I," Applied Scientific Research, vol. 2, no. 1, pp. 120-124, 1951.

[25] B. Gebhart, "Effects of viscous dissipation in natural convection," Journal of Fluid Mechanics, vol. 14, no. 2, pp. 225-232, 1962.

[26] P. V. S. N. Murthy and P. Singh, "Effect of viscous dissipation on a non-Darcy natural convection regime," International Journal of Heat and Mass Transfer, vol. 40, pp. 1251-1260, 1977.

[27] G. Tunc and Y. Bayazitoglu, "Heat transfer in microtubes with viscous dissipation," International Journal of Heat and Mass Transfer, vol. 44, no. 13, pp. 2395-2403, 2001.

[28] C. I. Cookey, A. Ogulub, and V. B. Omubo-Peppleb, "Influence of viscous dissipation and radiation on unsteady MHD free-convection flow past an infinite heated vertical plate in a porous medium with time-dependent suction," International Journal of Heat and Mass Transfer, vol. 46, no. 13, pp. 2305-2311, 2003.

[29] M. K. Partha, P. V. S. N. Murthy, and G. P. Rajasekhar, "Effect of viscous dissipation on the mixed convection heat transfer from an exponentially stretching surface," Heat and Mass Transfer, vol. 41, no. 4, pp. 360-366, 2005.

[30] F. S. Ibrahim, A. M. Elaiw, and A. A. Bakr, "Influence of viscous dissipation and radiation on unsteady MHD mixed convection flow of micropolar fluids," Applied Mathematics \& Information Sciences, vol. 2, pp. 143-162, 2008.

[31] M. Y. Malik, A. Hussain, T. Salahuddin, M. Awais, S. Bilal, and F. Khan, "Flow of sisko fluid over a stretching cylinder and heat transfer with viscous dissipation and variable thermal conductivity: A numerical study," AIP Advances, vol. 6, no. 4, Article ID 045118, 2016.

[32] A. Hussain, M. Y. Malik, T. Salahuddin, A. Rubab, and M. Khan, "Effects of viscous dissipation on MHD tangent hyperbolic fluid over a nonlinear stretching sheet with convective boundary conditions," Results in Physics, vol. 7, pp. 3502-3509, 2017.

[33] J. H. Merkin, "On dual solutions occurring in mixed convection in a porous medium," Journal of Engineering Mathematics, vol. 20, no. 2, pp. 171-179, 1986. 